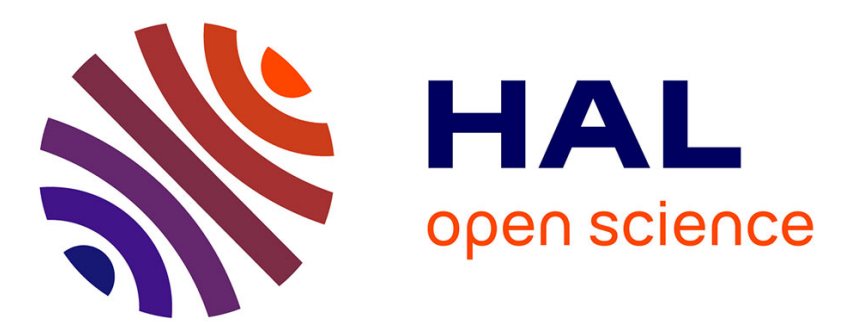

\title{
Fast Tracking of Poiseuille Trajectories in Navier-Stokes 2D Channel Flow
}

\author{
Jean-Michel Coron, Emmanuel Trélat, Rafael Vazquez
}

\section{To cite this version:}

Jean-Michel Coron, Emmanuel Trélat, Rafael Vazquez. Fast Tracking of Poiseuille Trajectories in Navier-Stokes 2D Channel Flow. CTS-HYCON Workshop, 2006, Paris, France. pp.699-720. hal00086480

\section{HAL Id: hal-00086480 \\ https://hal.science/hal-00086480}

Submitted on 18 Jul 2006

HAL is a multi-disciplinary open access archive for the deposit and dissemination of scientific research documents, whether they are published or not. The documents may come from teaching and research institutions in France or abroad, or from public or private research centers.
L'archive ouverte pluridisciplinaire HAL, est destinée au dépôt et à la diffusion de documents scientifiques de niveau recherche, publiés ou non, émanant des établissements d'enseignement et de recherche français ou étrangers, des laboratoires publics ou privés. 


\title{
Fast Tracking of Poiseuille Trajectories in Navier Stokes 2D Channel Flow
}

\author{
Rafael Vázquez ${ }^{\star}$, Emmanuel Trélat $^{\dagger}$ and Jean-Michel Coron $^{\ddagger}$ \\ * Department of Mechanical and Aerospace Eng. \\ Univ. of California San Diego, La Jolla, 92037 CA, USA \\ Email:rvazquez@ucsd.edu \\ $\dagger$ Univ. Paris-Sud, Lab. Math. \\ UMR 8628, 91405 Orsay Cedex, France \\ Email: Emmanuel.Trelat@math.u-psud.fr \\ ${ }^{\ddagger}$ Institut Universitaire de France and Univ. Paris-Sud, Lab. Math. \\ UMR 8628, 91405 Orsay Cedex, France \\ Email:Jean-Michel.Coron@math.u-psud.fr
}

ABSTRACT. We consider the problem of generating and tracking a trajectory between two arbitrary parabolic profiles of a periodic $2 D$ channel flow, which is linearly unstable for high Reynolds numbers. Also known as the Poisseuille flow, this problem is frequently cited as a paradigm for transition to turbulence. Our approach consists in generating an exact trajectory of the nonlinear system that approaches exponentially the objective profile. A boundary control law guarantees then that the error between the state and the trajectory decays exponentially in the $L^{2}$ norm. The result is first proved for the linearized Stokes equations, then shown to hold for the nonlinear Navier Stokes system.

RÉSUMÉ. A définir par la commande \resume $\{\ldots\}$

KEYWORDS: Distributed parameter systems, Partial differential equations, Tracking systems, Fourier analysis, Nonlinear control systems

MOTS-CLÉS : A définir par la commande \motscles $\{.$. 


\section{Introduction}

One of the few situations in which analytic expressions for solutions of the stationary flow field are available is the channel flow problem. Also known as the Poiseuille flow, this problem is frequently cited as a paradigm for transition to turbulence. Poiseuille flow requires an imposed external pressure gradient for being created and sustained [BAT 67]. The magnitude of the pressure gradient determines the value of the centerline velocity, which parameterizes the whole flow.

It is very well known that this solution goes linearly unstable for Reynolds numbers greater than the so-called critical Reynolds number, $R e_{C R} \approx 5772$ [SCH 01]. The problem of locally stabilizing the equilibrium has been considered by means of optimal control [HOG 03], and using the backstepping technique [V'05a]. Observers have been developed as well using dual methods [HOE 05, $\mathrm{V}^{\prime} 05 \mathrm{~b}$ ].

However, all prior works consider a constant pressure gradient and a developed flow which is already close to the desired solution. The problem of tracking time varying profiles generated by unsteady pressure gradients has, so far, not been considered from a control point of view. Stability for channel flow driven by unsteady pressure gradient has been previously studied [KER 82].

In this paper we consider the problem of moving the state from one Poiseuille equilibrium to another, following a pre-determined flow trajectory that should be "nice" in some sense. For example, we may wish to smoothly accelerate fluid at rest up to a given Reynolds number, probably over the critical value, avoiding transition to turbulence. The means at our disposal are the imposed pressure gradient and boundary control of the velocity field. We consider velocity actuation at one of the walls.

This is a problem of practical interest which, to the best of our knowledge, has not been solved or even been considered so far, since all control laws in the literature are designed for one given Poiseuille flow (fixed Reynolds number).

A possible solution for the problem would be to apply quasi-static deformation theory; this would require to modify the pressure gradient very slowly, and simultaneously gain-schedule a fixed Reynold number boundary controller like [V’05a] for tracking a (slowly) time varying trajectory, which in general would not be an exact solution of the system. This idea has been already used for moving between equilibria of a nonlinear parabolic equation [COR 04], or a wave equation [COR 05]. Other applications include the shallow water problem [COR 02] and the Couette-Taylor flow [SCH 05]. In this paper, however, we follow an alternative approach, finding an exact, fast trajectory of the system which is then stabilized by means of boundary control. The advantage of this approach is that it reaches the objective profile requiring substantially less time and control effort.

The organization of the paper is as follows. We begin stating the model in Section 2. In Section 3, we consider and solve the problem of generating an exact trajectory between two Poiseuille profiles. Then, in Section 4 we present the boundary control laws and our main results. We follow with Section 5, where we present the 


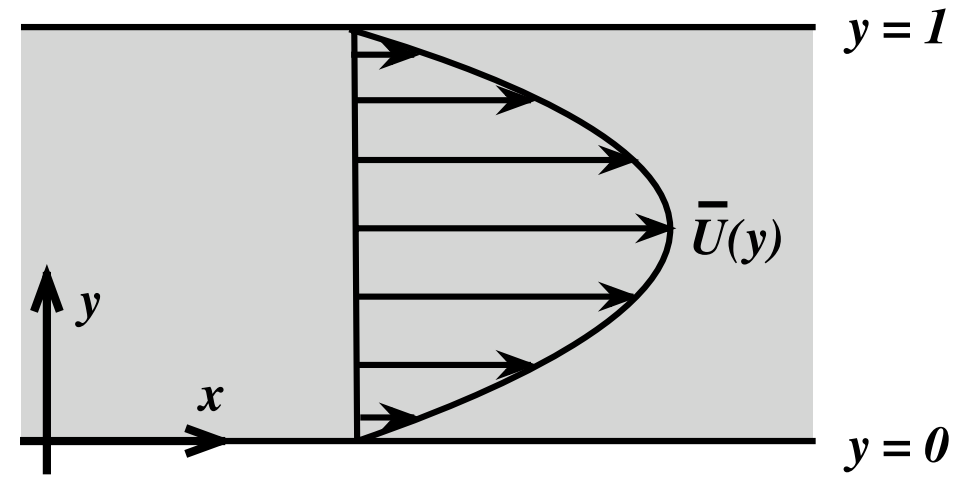

Figure 1. 2D Channel Flow with an equilibrium profile

mathematical framework required to solve the problem. Sections 6 and 7 are devoted to the proofs of the main results, in the linear and nonlinear case, respectively.

\section{Channel flow model}

We consider 2-D incompressible fluid filling a region $\Omega$ between two infinite planes separed from each other a distance $L$. The exact setting is depicted in Fig. 1 , in which an example equilibrium profile is shown. Define $U_{c}$ as a velocity scale, where $U_{c}$ is the maximum centerline velocity, $\rho$ and $\nu$ as the density and the kinematic viscosity of the fluid, respectively, and the Reynolds number, $R e$, as $R e=U_{c} h / \nu$. Then, using $L, L / U_{c}$ and $\rho \nu U_{c} / L$ as length, time and pressure scales respectively, we can write the nondimensional 2-D Navier-Stokes equations as follows,

$$
\begin{aligned}
& u_{t}=\frac{\triangle u}{R e}-p_{x}-u u_{x}-v u_{y}, \\
& v_{t}=\frac{\triangle v}{R e}-p_{y}-u v_{x}-v v_{y},
\end{aligned}
$$

where $u$ is the streamwise velocity, $v$ the wall-normal velocity, and $p$ the pressure, with boundary conditions

$$
\begin{aligned}
& u(t, x, 0)=v(t, x, 0)=0, \\
& u(t, x, 1)=U(t, x), \\
& v(t, x, 1)=V(t, x) .
\end{aligned}
$$

In (4) and (5), $U$ and $V$ are the actuators located at the upper wall, which can be actuated independently for each $x$. Additionally we consider incompressible fluid, so the velocity field must verify in $\Omega$ the divergence-free condition

$$
u_{x}+v_{y}=0 .
$$


In this nondimensional coordinates, $\Omega$ can be defined as

$$
\Omega=\left\{(x, y) \in \mathbb{R}^{2}: 0 \leq y \leq 1\right\},
$$

with boundary $\partial \Omega=\partial \Omega_{0} \cup \partial \Omega_{1}$, where

$$
\partial \Omega_{i}=\left\{(x, y) \in \mathbb{R}^{2}: y=i\right\} .
$$

$\partial \Omega_{0}$ will be referred as the uncontrolled boundary and $\partial \Omega_{1}$ as the controlled boundary.

\section{Trajectory generation and control objective}

The stationary family of solutions of (1)-(5) is the Poiseuille family of parabolic profiles, $\mathcal{P}^{\delta}$, which is described by a single parameter $\delta$ (the maximum centerline velocity) in the following way

$$
\mathcal{P}^{\delta}=\left(u^{\delta}, v^{\delta}, p^{\delta}\right)=\left(4 \delta y(1-y), 0,-\frac{8 \delta}{R e} x\right) .
$$

Note that the velocity actuation at the wall is zero for $\mathcal{P}^{\delta}$, since both $u^{\delta}$ and $v^{\delta}$ are zero at the boundaries. The pressure gradient $p_{x}^{\delta}=-\frac{8 \delta}{R e}$ must be externally sustained for (9) to be a stationary solution [BAT 67].

Our first task is, given $\delta_{0}$ and $\delta_{1}$, generate an unsteady trajectory path $\Theta(t)=$ $(u(t), v(t), p(t))$, where space dependence is omitted for clarity, connecting $\mathcal{P}^{\delta_{0}}$ to $\mathcal{P}^{\delta_{1}}$. We assume $\delta_{0}=0$ and $\delta_{1}=1$ for simplicity. Other values may be considered using the same tools.

Consider the trajectory $\Theta^{q}(t)$ defined as

$$
\begin{aligned}
\Theta^{q}(t) & =\left(u^{q}(t), v^{q}(t), p^{q}(t)\right) \\
& =(g(t, y), 0, x q(t)),
\end{aligned}
$$

where $q$ is the chosen external pressure gradient. Then, by substitution we see that (10) verifies (1)-(5) if

$$
g_{t}=\frac{g_{y y}}{R e}-q .
$$

Since $\mathcal{P}^{0} \equiv 0$, we set $\Theta^{q}(0)=0$, which implies $g(0, y)=q(0)=0$. We impose $g(t, 0)=g(t, 1)=0$ so no velocity control effort is needed to steer the trajectory, only to stabilize it. Given these initial-boundary data, choosing $q$ completely determines $g$ from (11) and consequently $\Theta^{q}(t)$, so $q(t)$ parameterizes $\Theta^{q}(t)$.

Choosing $q(t)$ as

$$
q(t)=\frac{8}{R e}\left(1-\mathrm{e}^{-c t}\right),
$$

with $c>0$ a design parameter, then $q(0)=0$ and $\lim _{t \rightarrow \infty} q(t)=-8 / R e$. 
Introducing (12) in (11), we can solve for $g$ analytically. Supposing $c \neq \pi^{2}(2 m+$ $1)^{2} / \operatorname{Re}$ for $m \in \mathbb{Z}, g$ is then

$$
\begin{aligned}
g= & 16 \sum_{m=0}^{m=\infty} \frac{\sin ((2 m+1) \pi y)}{(2 m+1)^{3} \pi^{3}}\left[1-\mathrm{e}^{-\frac{\pi^{2}(2 m+1)^{2}}{R e} t}\right. \\
& \left.-\frac{\mathrm{e}^{-c t}-\mathrm{e}^{-\frac{\pi^{2}(2 m+1)^{2}}{R e} t}}{1-\frac{c R e}{\pi^{2}(2 m+1)^{2}}}\right] .
\end{aligned}
$$

As time grows, $g(t, y)$ goes exponentially to its steady state

$$
\begin{aligned}
\lim _{t \rightarrow \infty} g(t, y) & =16 \sum_{m=0}^{m=\infty} \frac{\sin ((2 m+1) \pi y)}{(2 m+1)^{3} \pi^{3}} \\
& =4 y(1-y) .
\end{aligned}
$$

It can be proved as well ${ }^{1}$ that $g(t, y)$ is analytic on its domain of definition and verifies

$$
\begin{aligned}
|g(t, y)| & \leq 1 \\
\left|g_{y}(t, y)\right| & \leq 4
\end{aligned}
$$

In Figure 2 we represent $g$, computed numerically from (11), for $c=1, R e=1$.

It follows that $\Theta^{q}(t)$ is a solution of the trajectory generation problem, since its components are smooth (in fact analytic) and solve (1)-(5), and additionaly, we have that $\Theta^{q}(0)=\mathcal{P}^{0}$ and $\lim _{t \rightarrow \infty} \Theta^{q}(t)=\mathcal{P}^{1}$, so $\Theta^{q}(t)$ connects the chosen Poiseuille profiles $^{2}$.

Using (10), we define the error variables as

$$
\begin{aligned}
(\tilde{u}, \tilde{v}, \tilde{p}) & =(u, v, p)-\Theta^{q}(t) \\
& =(u-g(t, y), v, p-x q(t)) .
\end{aligned}
$$

The error variables verify the following equations,

$$
\begin{aligned}
\tilde{u}_{t}= & \frac{\triangle \tilde{u}}{R e}-\tilde{p}_{x}-\tilde{u} \tilde{u}_{x}-\tilde{v} \tilde{u}_{y}-g(t, y) \tilde{u}_{x} \\
& -g_{y}(t, y) \tilde{v}, \\
\tilde{v}_{t}= & \frac{\triangle \tilde{v}}{R e}-\tilde{p}_{y}-\tilde{u} \tilde{v}_{x}-\tilde{v} \tilde{v}_{y}-g(t, y) \tilde{v}_{x},
\end{aligned}
$$

1. Using the maximum principle and other heat equation properties [EVA 98].

2. Reaching $\mathcal{P}^{1}$ only after an infinitely long time, however by construction through rapidly decaying exponentials, $\Theta^{q}$ closely approaches $\mathcal{P}^{1}$ after a short time, as shown in Fig. 2. In this sense, we consider $\Theta^{q}$ a fast trajectory. 


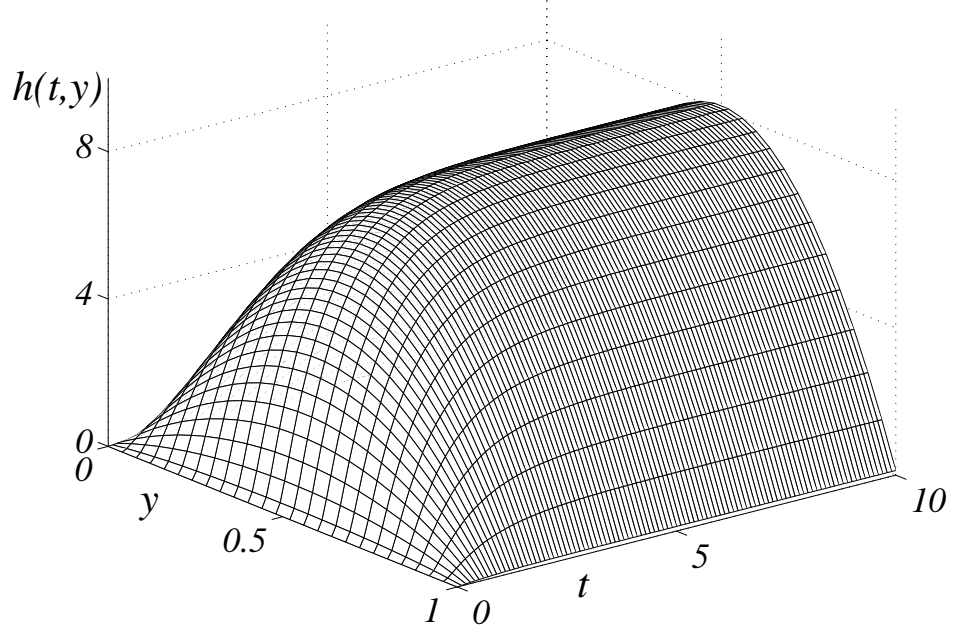

Figure 2. Evolution of $g(t, y)$ for $c=1, R e=1$.

and the same boundary conditions and divergence-free condition as before. Our new control objetive is to stabilize the equilibrium at the origin in (18)-(19) by means of $U$ and $V$. Achieving that implies, considering (17), that the trajectory $\Theta^{q}$ is stabilized.

Linearizing (18)-(19) around $\Theta^{q}$, and dropping tildes, we obtain the unsteady Stokes equations

$$
\begin{aligned}
u_{t} & =\frac{\triangle u}{R e}-p_{x}-g(t, y) u_{x}-g_{y}(t, y) v \\
v_{t} & =\frac{\triangle v}{R e}-p_{y}-g(t, y) v_{x}
\end{aligned}
$$

with boundary conditions

$$
\begin{aligned}
& u(t, x, 0)=v(t, x, 0)=0, \\
& u(t, x, 1)=U(t, x), \\
& v(t, x, 1)=V(t, x) .
\end{aligned}
$$

We consider first the problem of stabilizing the origin of (20)-(21), and later show how its solution stabilizes, even though only locally ${ }^{3}$, the origin of (18)-(19).

3. Local stabilization of the origin of (18)-(19) suffices, since we assume the initial data are zero, i.e. the velocity field starts at the origin itself. 


\section{Main results}

Consider the following control laws.

The controller $V(t, x)$ is a dynamic controller, found as the unique solution of the following forced parabolic equation

$$
\begin{aligned}
V_{t}= & \frac{V_{x x}}{R e}-\sum_{0<|n|<M} \int_{-h}^{h} \mathrm{e}^{i \gamma_{n}(\xi-x)} \\
& \times\left[2 i \int_{0}^{1} g_{y}(t, \eta) \cosh \left(\gamma_{n}(1-\eta)\right) V(\tau, \xi, \eta) d \eta\right. \\
& \left.-i \frac{u_{y}(t, x, 0)-u_{y}(t, x, 1)}{R e}\right] d \xi
\end{aligned}
$$

which we initialize at zero, whereas the control law $U$ is

$$
\begin{aligned}
U= & \sum_{0<|n|<M} \int_{-h}^{h} \int_{0}^{1} \mathrm{e}^{i \gamma_{n}(\xi-x)} K_{n}(t, 1, \eta) \\
& \times u(t, \xi, \eta) d \eta d \xi
\end{aligned}
$$

where $M=\frac{2 h \sqrt{R e}}{\pi}$ and $\gamma_{n}=\pi n / h . K_{n}$ in (26) is the solution, for each $n$, of the following kernel equation ${ }^{4}$

$$
\begin{aligned}
K_{n t}= & \frac{1}{R e}\left(K_{n y y}-K_{n \eta \eta}\right)-\lambda_{n}(t, \eta) K_{n}+f(y, \eta) \\
& -\int_{\eta}^{y} f(\xi, \eta) K_{n}(t, y, \xi) d \xi
\end{aligned}
$$

a linear partial integro-differential equation in the region $\Gamma=(t, y, \eta) \in(0, \infty) \times \mathcal{T}$, where $\mathcal{T}=\left\{(y, \eta) \in \mathbb{R}^{2}: 0 \leq \eta \leq y \leq 1\right\}$, with boundary conditions:

$$
\begin{aligned}
K_{n}(t, y, y)= & -\operatorname{Re}\left(\lambda(y) \frac{y}{2}+h_{n}(0)\right) \\
K_{n}(t, y, 0)= & \operatorname{Re}\left[\int_{0}^{y} h_{n}(\sigma) K_{n}(t, y, \sigma) d \sigma\right. \\
& -h(y)]
\end{aligned}
$$

4. See Proposition 6.1 in Section 6 regarding the solvability of (27)-(29). 
and where the functions that appear in (27)-(29) are

$$
\begin{aligned}
\lambda_{n}(t, y)= & i \gamma_{n} g(t, y), \\
f_{n}(t, y, \eta)= & -i \gamma_{n}\left[g_{y}(t, y)+2 \gamma_{n} \int_{\eta}^{y} g_{y}(t, \sigma)\right. \\
& \left.\times \sinh \left(\gamma_{n}(y-\sigma)\right) d \sigma\right], \\
h_{n}(y)= & \frac{\gamma_{n}}{R e}\left[\frac{\cosh \left(\gamma_{n}(1-y)\right)}{\sinh \gamma_{n}}\right. \\
& \left.-\frac{\left.\cosh \left(\gamma_{n} y\right)\right)}{\sinh \gamma_{n}}\right] .
\end{aligned}
$$

We state now our results.

Theorem 4.1 For any Reynolds number, the equilibrium $u \equiv v \equiv 0$ of Stokes system (20)-(24) with control laws (25)-(26) is globally exponentially stable in the $L^{2}$ norm, i.e., if $\mathbf{w}=(u, v)$, there exist numbers $C_{1}(R e), C_{2}(R e)>0$ such that for $t \geq 0$,

$$
\|\mathbf{w}(t)\| \leq C_{1} \mathrm{e}^{-C_{2} t}\|\mathbf{w}(0)\| .
$$

The result above is valid for any initial condition. If we consider the nonlinear terms, we obtain just local stability.

Theorem 4.2 For any Reynolds number, the equilibrium $u \equiv v \equiv 0$ of the Navier Stokes system (18)-(19) with boundary conditions (22)-(24) and control laws (25)(26) is locally exponentially stable in the $L^{2}$ norm, i.e., if $\mathbf{w}=(u, v)$, there exist numbers $\epsilon(R e), C_{1}(R e), C_{2}(R e)>0$ such that if $\|\mathbf{w}(0)\|<\epsilon$, and for $t \geq 0$,

$$
\|\mathbf{w}(t)\| \leq C_{1} \mathrm{e}^{-C_{2} t}\|\mathbf{w}(0)\| .
$$

From the results of Section 3 and Theorem 4.2, the next result, which solves the problem, follows.

Theorem 4.3 For any Reynolds number, $\Theta^{q}(t)$ defined by (10)-(12) is a solution of system (1)-(5), with imposed pressure gradient (12), and control laws (25)-(32) expressed in the error variables (17). Moreover, this solution is locally exponentially stable in the $L^{2}$ norm. In particular, if the state is initialized close enough to rest, it closely follows $\Theta^{q}(t)$ and approaches the steady equilibrium $\mathcal{P}^{1}$ exponentially fast.

REMARK. - Even though the controller (25)-(32) looks rather involved, it is not hard to compute and implement. One has to solve a finite set of linear PIDE equations (27)-(29) for computing the $K_{n}$ 's, which can be done fast and efficiently compared, 
for example, with $\mathrm{LQR}$ - where nonlinear time dependent Ricatti equations appear. See [SMY 04] for a numerical comparison between LQR and backstepping.

REMARK. - This result can be extended in a number of ways. An output feedback design is possible applying a dual backstepping observer methodology [SMY 05a, $\mathrm{V}^{\prime} 05 \mathrm{~b}$, only requiring boundary measurements of pressure and skin friction. A 3D channel flow, periodic in two directions, is also tractable, adding some refinements which include actuation of the spanwise velocity at the wall. Stability in the $H^{1}$ norm can be obtained as well. Details will appear in an upcoming publication.

REMARK. - Averaging in $x$ expression (25) it can be deduced that the mean component of $V$ is zero, thus the physical constraint of zero net flux is verified.

The next sections are devoted to proving the results, introducing precise definition of the norms and spaces for the velocity field, explaining the control design method, and studying the solvability of equation (27)-(29).

\section{Mathematical preliminaries}

In this section we present the framework that we use to solve the stabilization problem.

\subsection{Periodic function spaces}

Since $\Omega$ is unbounded, we assume that the velocity field $(u, v)$ and the pressure $p$ are periodic in $x$ with some period $2 h>0$ [TEM 84]. This requires for consistency that $U$ and $V$ are periodic with the same period; this is already verified by expressions (25)-(26). In this setting, $\Omega$ and its boundary are identified with

$$
\begin{aligned}
\Omega_{h} & =\{(x, y) \in \Omega:-h \leq x \leq h\}, \\
\partial \Omega_{h i} & =\left\{(x, y) \in \partial \Omega_{i}:-h \leq x \leq h\right\} .
\end{aligned}
$$

Let $L^{2}\left(\Omega_{h}\right)$ be the usual Lebesgue space of square-integrable functions, endowed with the scalar product

$$
(\phi, \psi)_{L^{2}\left(\Omega_{h}\right)}=\int_{-h}^{q} \int_{0}^{1} \phi(x, y) \psi(x, y) d y d x
$$

Define then $L_{h}^{2}(\Omega)=L^{2}\left(\Omega_{h}\right)$, where now

$$
(\phi, \psi)_{L_{h}^{2}(\Omega)}=\left(\left.\phi\right|_{\Omega_{h}},\left.\psi\right|_{\Omega_{h}}\right)_{L^{2}\left(\Omega_{h}\right)} .
$$




\subsection{Fourier series expansion}

Given a function $\phi$ we define the sequence of its complex Fourier coefficients $\left(\phi_{n}(y)\right)_{n \in \mathbb{Z}}$ as

$$
\phi_{n}(y)=\frac{1}{2 h} \int_{-h}^{h} \phi(x, y) \mathrm{e}^{\frac{i n \pi}{h} x} d x, n \in \mathbb{Z} .
$$

We will write simply $\phi_{n}$ in the sequel. It can be shown that if in $\phi \in L^{2}\left(\Omega_{h}\right)$, then (39) is well defined and $\phi_{n}$ is in the (complex valued) $\ell^{2} \times L^{2}(0,1)$ space, i.e.,

$$
\sum_{n \in \mathbb{Z}} \int_{0}^{1}\left|\phi_{n}(y)\right|^{2} d y<\infty .
$$

One can recover $\phi$ by writting its Fourier series,

$$
\phi(x, y)=\sum_{n \in \mathbb{Z}} \phi_{n}(t, y) \mathrm{e}^{-\frac{i n \pi}{h} x} .
$$

Equation (41) always yields a $L^{2}\left(\Omega_{h}\right)$ function, if $\phi_{n} \in \ell^{2} \times L^{2}(0,1)$.

One important results is Parseval's formula,

$$
(\phi, \psi)_{L^{2}\left(\Omega_{h}\right)}=\left(\phi_{n}, \psi_{n}\right)_{\ell^{2} \times L^{2}(0,1)}
$$

where the $\ell^{2} \times L^{2}(0,1)$ scalar product is

$$
\left(\phi_{n}, \psi_{n}\right)_{\ell^{2} \times L^{2}(0,1)}=\sum_{n \in \mathbb{Z}} \int_{0}^{1} \phi_{n}(y) \bar{\psi}_{n}(y) d y,
$$

and where the bar denotes the complex conjugate.

In the sequel we omit the subindexes in the sums when referring to $\mathbb{Z}$, and in norms and scalar products when the corresponding space (usually $L^{2}$ ) is clear from the context.

Using (42), and given $\psi$ in $L^{2}\left(\Omega_{h}\right)$, we can compute its norm by computing its Fourier coefficients $\psi_{n}$. Then,

$$
\|\psi\|_{L^{2}\left(\Omega_{h}\right)}^{2}=\|\psi\|_{\ell^{2} \times L^{2}(0,1)}^{2}=\sum\left\|\psi_{n}\right\|_{L^{2}(0,1)}^{2}
$$

where

$$
\left\|\psi_{n}\right\|_{L^{2}(0,1)}^{2}=\int_{0}^{1}\left|\psi_{n}(y)\right|^{2} d y
$$

\section{3. $H^{1}$ spaces}

We define the space $H_{h}^{1}(\Omega)$ as

$$
H_{h}^{1}(\Omega)=\left\{\left.f\right|_{\Omega_{h}} \in H^{1}\left(\Omega_{h}\right),\left.f\right|_{x=-h}=\left.f\right|_{x=h} \text { a.e. }\right\} .
$$


The $H^{1}$ norm is defined as

$$
\|\phi\|_{H_{h}^{1}(\Omega)}^{2}=\|\phi\|_{L_{h}^{2}(\Omega)}^{2}+\left\|\phi_{y}\right\|_{L_{h}^{2}(\Omega)}^{2}+\left\|\phi_{x}\right\|_{L_{h}^{2}(\Omega)}^{2}
$$

or in terms of the Fourier coefficients

$$
\begin{aligned}
\|\phi\|_{H_{h}^{1}(\Omega)}^{2}= & \sum\left[\left(1+4 \pi^{2} n^{2}\right)\left\|\phi_{n}\right\|_{L^{2}(0,1)}^{2}\right. \\
& \left.+\left\|\phi_{n y}\right\|_{L^{2}(0,1)}^{2}\right]
\end{aligned}
$$

We state the following lemma:

Lemma 5.1 Suppose that $\phi \in H_{h}^{1}(\Omega)$ such that $\left.\phi\right|_{\partial \Omega_{0}} \equiv 0$, and $\psi \in L_{h}^{2}(\Omega)$. Then:

$$
\left(\phi^{2}, \psi^{2}\right)_{L_{h}^{2}(\Omega)} \leq\left\|\phi_{y}\right\|_{L_{h}^{2}(\Omega)}^{2}\|\psi\|_{L_{h}^{2}(\Omega)}^{2} .
$$

Proof Using Fourier coefficients:

$$
\begin{aligned}
\left(\phi^{2}, \psi^{2}\right)_{L_{h}^{2}(\Omega)} & =\sum\left(\phi_{n}^{2}, \psi_{n}^{2}\right)_{L^{2}(0,1)} \\
& =\sum \int_{0}^{1} \phi_{n}^{2}(y) \bar{\psi}_{n}^{2}(y) d y .
\end{aligned}
$$

Now, since $\phi \in H_{h}^{1}(\Omega)$, then $\phi_{n} \in H^{1}(0,1)$, and since $\left.\phi\right|_{\partial \Omega_{0}} \equiv 0$, this implies that $\left.\phi_{n}\right|_{y=0} \equiv 0$. Then

$$
\phi_{n}(y)^{2} \leq\left\|\phi_{n y}\right\|_{L^{2}(0,1)}^{2}
$$

and taking supremum:

$$
\left\|\phi_{n}\right\|_{L_{\infty}}^{2} \leq\left\|\phi_{n y}\right\|_{L^{2}(0,1)}^{2}
$$

so then

$$
\begin{aligned}
\int_{0}^{1} \phi_{n}^{2}(y) \bar{\psi}_{n}^{2}(y) d y & \leq\left\|\phi_{n}\right\|_{L_{\infty}}^{2} \int_{0}^{1}\left|\psi_{n}\right|^{2}(y) d y \\
& \leq\left\|\phi_{n y}\right\|_{L^{2}(0,1)}^{2}\left\|\psi_{n}\right\|_{L^{2}(0,1)}^{2}
\end{aligned}
$$

therefore

$$
\begin{aligned}
\left(\phi^{2}, \psi^{2}\right)_{L_{h}^{2}(\Omega)} & \leq \sum\left\|\phi_{n y}\right\|_{L^{2}(0,1)}^{2}\left\|\psi_{n}\right\|_{L^{2}(0,1)}^{2} \\
& \leq\left(\sum\left\|\phi_{n y}\right\|_{L^{2}(0,1)}^{2}\right)\left(\sum\left\|\psi_{n}\right\|_{L^{2}(0,1)}^{2}\right) \\
& =\left\|\phi_{y}\right\|_{L_{h}^{2}(\Omega)}^{2}\|\psi\|_{L_{h}^{2}(\Omega)}^{2},
\end{aligned}
$$

in which we have used that for two sequences $a_{n}, b_{n}$ in $\ell_{1}$, one has

$$
\left(a_{n}, b_{n}\right)_{\ell_{2}} \leq\left\|a_{n}\right\|_{\ell_{2}}\left\|b_{n}\right\|_{\ell_{2}} \leq\left\|a_{n}\right\|_{\ell_{1}}\left\|b_{n}\right\|_{\ell_{1}} \text {. }
$$

This fact follows from the Cauchy-Schwartz inequality and noting that $\|\cdot\|_{\ell_{2}} \leq\|\cdot\|_{\ell_{1}}$. 


\subsection{Spaces for the velocity field}

Calling $\mathbf{w}=(u, v)$, we define

$$
H_{0 h}(\Omega)=\left\{\mathbf{w} \in\left[L_{h}^{2}(\Omega)\right]^{2}: \nabla \cdot \mathbf{w}=0,\left.\mathbf{w}\right|_{\partial \Omega_{0}}=0\right\}
$$

and

$$
H_{0 h}^{1}(\Omega)=H_{0 h}(\Omega) \cap\left[H_{h}^{1}(\Omega)\right]^{2},
$$

endowed with the scalar product of, respectively, $\left[L_{h}^{2}(\Omega)\right]^{2}$ and $\left[H_{h}^{1}(\Omega)\right]^{2}$. These are the spaces for the velocity field and where Theorems 4.1 and 4.2 have to be considered.

\subsection{Transformations of $L^{2}$ functions}

The following definitions establish facts and notation useful for our solution, based on the backstepping method [SMY 04]. This method consists in finding an invertible transformation of the original variables into others whose stability properties are easy to establish. We study the kind of transformations that appear in the method.

Definition 5.1 Given complex valued functions $f \in L^{2}(0,1)$ and $K \in L^{\infty}(\mathcal{T})$, we define the transformed variable $g=(\mathrm{I}-K) f$, where the operator $K f$ is defined as

$$
K f=\int_{0}^{y} K(y, \eta) f(\eta) d \eta,
$$

i.e. a Volterra operator. We call $\mathrm{I}-K$ the direct transformation with kernel $K$. Now, if there exists a function $L \in L^{\infty}(\mathcal{T})$ such that $f=(\mathrm{I}+L) g$, then we say that the transformation is invertible, and we call $\mathrm{I}+L$ the inverse transformation, and $L$ the inverse kernel (or the inverse of $K$ ).

The following result is immediate from the theory of Volterra integral equations [HOC 73].

Proposition 5.1 For $K \in L^{\infty}(\mathcal{T})$, the transformation $\mathrm{I}-K$ is always invertible. Moreover, $L$ is related to $K$ by

$$
\begin{aligned}
L(y, \eta) & =K(y, \eta)+\int_{\eta}^{y} K(y, \sigma) L(\sigma, \eta) d \sigma \\
& =K(y, \eta)+\int_{\eta}^{y} L(y, \sigma) K(\sigma, \eta) d \sigma .
\end{aligned}
$$

The following results also holds:

Proposition 5.2 If $f \in L^{2}(0,1)$ then $g=(\mathrm{I}-K) f$ is in $L^{2}(0,1)$. Similarly, if $g \in L^{2}(0,1)$ then $f=(\mathrm{I}+L) g$ is in $L^{2}(0,1)$. Moreover,

$$
\begin{aligned}
& \|g\|^{2} \leq\left(1+\|K\|_{\infty}\right)^{2}\|f\|^{2}, \\
& \|f\|^{2} \leq\left(1+\|L\|_{\infty}\right)^{2}\|g\|^{2} .
\end{aligned}
$$


Proof Immediate from calculating the $L^{2}$ norm on the transformed variable in Definition 5.1, and then using Cauchy-Schwartz inequality repeatedly.

Proposition 5.2 allows to define a norm equivalent to the $L^{2}$ norm,

$$
\|f\|_{K L^{2}(0,1)}^{2}=\|(\mathrm{I}-K) f\|_{L^{2}(0,1)}^{2}=\|g\|_{L^{2}(0,1)}^{2} .
$$

For $\mathcal{C}^{1}(\mathcal{T})$ kernels $K$ and $L$, one has an equivalent version of Proposition 5.1 and Proposition 5.2, allowing to define a $K H^{1}(0,1)$ norm, which is equivalent to the $H^{1}(0,1)$ norm.

\subsection{Transformations of the velocity field}

We define transformations of functions in $H_{0 h}(\Omega)$.

Definition 5.2 Suppose we have a finite set $A=\left\{a_{1}, \ldots, a_{j}\right\} \subset \mathbb{Z}$ and $\mathcal{K}=\left(K_{n}(y, \eta)\right)_{n \in A}$ is a family of $L^{\infty}(\mathcal{T})$ kernels. Then, for $\mathbf{w}=(u, v), \mathbf{w} \in H_{0 h}(\Omega)$, one defines the transformed variable $\omega=(\alpha, \beta)=(\mathrm{I}-\mathcal{K}) \mathbf{w}$, through its Fourier components,

$$
\omega_{n}=\left\{\begin{array}{c}
\left(\left(\mathrm{I}-K_{n}\right) u_{n}, 0\right) n \in A \\
\mathbf{w}_{n}, \text { otherwise. }
\end{array}\right.
$$

The inverse transformation, $\mathbf{w}=(I+\mathcal{L}) \omega$, is defined as

$$
\mathbf{w}=\left\{\begin{array}{c}
\left(\left(\mathrm{I}+L_{n}\right) \alpha_{n}, \hat{L}_{n} \alpha_{n}\right) n \in A \\
\omega_{n}, \text { otherwise }
\end{array}\right.
$$

where the new operator $\hat{L}_{n}$ is defined as:

$$
\hat{L}_{n} f=-\pi i \frac{n}{h} \int_{0}^{y}\left(f(\eta)+\int_{0}^{\eta} L(\eta, \sigma) f(\sigma) d \sigma\right) d \eta .
$$

It is straightforward that the inverse is correctly defined. We only need to check the second component of $\mathbf{w}$ in (64) when $n \in A$, which is

$$
\hat{L}_{n} \alpha_{n}=-\pi i \frac{n}{h} \int_{0}^{y}\left(\alpha_{n}(\eta)+\int_{0}^{\eta} L(\eta, \sigma) \alpha_{n}(\sigma) d \sigma\right) d \eta,
$$

and then substituting the definition of $\alpha_{n}$ from the direct transformation, and after some manipulation,

$$
\begin{aligned}
\hat{L}_{n} \alpha_{n}= & -\pi i \frac{n}{h} \int_{0}^{y}\left(u_{n}(\eta)-\int_{0}^{\eta}\left[K_{n}(\eta, \sigma)-L_{n}(\eta, \sigma)\right.\right. \\
& \left.\left.+\int_{\sigma}^{\eta} L_{n}(\eta, \delta) K_{n}(\delta, \sigma) d \delta\right] \times u_{n}(\delta) d \delta\right) d \eta
\end{aligned}
$$


where the expression in brackets is zero by Proposition 5.1. Then,

$$
\hat{L}_{n} \alpha_{n}=-\pi i \frac{n}{h} \int_{0}^{y} u_{n}(\eta) d \eta
$$

and since the divergence-free condition in Fourier space is $\pi i \frac{n}{h} u_{n}+v_{n y}=0$ and $v_{n}(0)=0$ one gets that

$$
\hat{L}_{n} \alpha_{n}=\int_{0}^{y} v_{n y}(\eta) d \eta=v_{n}(y)-v_{n}(0)=v_{n}(y) .
$$

This way, even though the second component of the velocity is apparently lost in the direct transformation, it can be recovered and the transformation is still invertible. Using a similar argument as in Proposition 5.2,

$$
\begin{aligned}
\|\omega\|_{H_{0 h}(\Omega)}^{2} & \leq\left(1+\|\mathcal{K}\|_{\infty}\right)^{2}\|\mathbf{w}\|_{H_{0 h}(\Omega)}^{2}, \\
\|\mathbf{w}\|_{H_{0 h}(\Omega)}^{2} & \leq\left(1+N^{2}\right)\left(1+\|\mathcal{L}\|_{\infty}\right)^{2}\|\omega\|_{H_{0 h}(\Omega)}^{2},
\end{aligned}
$$

where $N=\max _{n \in A}\left\{\pi \frac{n}{h}\right\}$, and

$$
\begin{aligned}
\|\mathcal{K}\|_{\infty} & =\max _{n \in A}\left\{\left\|K_{n}\right\|_{\infty}\right\}, \\
\|\mathcal{L}\|_{\infty} & =\max _{n \in A}\left\{\left\|L_{n}\right\|_{\infty}\right\} .
\end{aligned}
$$

This allows the definition of a norm, as in (62), equivalent to the $H_{0 h}(\Omega)$, that we call $\mathcal{K} H_{0 h}(\Omega)$. For $\mathcal{C}^{1}(\mathcal{T})$ kernel families one can define as well a $\mathcal{K} H_{0 h}^{1}(\Omega)$ norm, equivalent to the regular $H_{0 h}^{1}(\Omega)$ norm.

REMARK. - All previous results hold for transformation kernels depending on time, as long as they are uniformly bounded on the time interval (finite or infinite) considered (see Proposition 6.1 for such a statement).

\section{Proof of theorem 4.1}

Equations (20)-(21) in Fourier space are

$$
\begin{aligned}
u_{n t} & =\frac{\triangle_{n} u_{n}}{R e}-i \gamma_{n}\left(p_{n}+g(t, y) u_{n}\right)-g_{y}(t, y) v_{n} \\
v_{n t} & =\frac{\triangle_{n} v_{n}}{R e}-p_{n y}-i \gamma_{n} g(t, y) v_{n},
\end{aligned}
$$

where $\triangle_{n}=\partial_{y y}-\gamma_{n}^{2}$ has been introduced for simplifying the expressions. The boundary conditions are

$$
\begin{aligned}
& u_{n}(t, 0)=v_{n}(t, 0)=0 \\
& u_{n}(t, 1)=U_{n}(t), \\
& v_{n}(t, 1)=V_{n}(t),
\end{aligned}
$$


and the divergence-free condition becomes

$$
\gamma_{n} u_{n}+v_{n y}=0 .
$$

From (74)-(75) an equation for the pressure can be derived,

$$
p_{n y y}-\gamma_{n}^{2} p_{n}=-2 i \gamma_{n} g_{y}(t, y) v_{n},
$$

with boundary conditions obtained from evaluating (75) at the boundaries and using (77)-(78),

$$
\begin{aligned}
& p_{n y}(0, t)=-i \gamma_{n} \frac{u_{n y}(0, t)}{R e} \\
& p_{n y}(1, t)=-i \gamma_{n} \frac{u_{n y}(1, t)}{R e}-\dot{V}_{n}-\gamma_{n}^{2} \frac{V_{n}}{R e} .
\end{aligned}
$$

Equations for different $n$ are uncoupled due to linearity and spatial invariance, allowing separate consideration for each mode $n$. Most modes, which we refer to as

uncontrolled, are naturally stable and thus left without control. A finite set of modes, called controlled, are unstable and require control.

\subsection{Uncontrolled modes}

These are $n=0$ and large modes that verify $|n| \geq M$.

6.1.1. $n=0$ (mean velocity field)

From (79), $v_{0} \equiv 0 . u_{0}$ verifies

$$
u_{0 t}=\frac{u_{0 y y}}{R e},
$$

with $u_{0}(0)=u_{0}(1)=0$. The following estimate holds:

$$
\frac{d}{d t}\left\|u_{0}(t)\right\|_{L^{2}(0,1)}^{2} \leq \mathrm{e}^{-\frac{2}{R e} t}\left\|u_{0}(0)\right\|_{L^{2}(0,1)}^{2} .
$$

\subsubsection{Modes for large $|n|$}

If $\mathbf{w}_{n}=\left(u_{n}, v_{n}\right)$, then, considering no control $\left(V_{n}=U_{n}=0\right)$ :

$$
\begin{aligned}
\frac{d}{d t}\left\|\mathbf{w}_{n}\right\|^{2}= & -2 \frac{\left\|\mathbf{w}_{n y}\right\|^{2}}{R e}-2 \gamma_{n}^{2} \frac{\left\|\mathbf{w}_{n}\right\|^{2}}{R e}-\left(g_{y} u_{n}, v_{n}\right)-\left(g_{y} v_{n}, u_{n}\right) \\
& -\left(u_{n}, i \gamma_{n} p_{n}\right)-\left(i \gamma_{n} p_{n}, u_{n}\right)-\left(v_{n}, p_{n y}\right)-\left(p_{n y}, v_{n}\right) .
\end{aligned}
$$

Consider the pressure terms like those in the last two lines of (85). Using the divergence free condition $\gamma_{n} u_{n}+v_{n y}=0$, and integration by parts,

$$
-\left(u_{n}, i \gamma_{n} p_{n}\right)=-\left(v_{n y}, p_{n}\right)=\left(v_{n}, p_{n y}\right) .
$$


Therefore, the pressure terms in (85) cancel each other. Then, using Young's inequality with the remaining terms,

$$
\frac{d}{d t}\left\|\mathbf{w}_{n}\right\|^{2} \leq-2 \frac{\left\|\mathbf{w}_{n y}\right\|^{2}}{R e}-2 \gamma_{n}^{2} \frac{\left\|\mathbf{w}_{n}\right\|^{2}}{R e}+\left\|g_{y}\right\|_{L_{\infty}}\left\|\mathbf{w}_{n}\right\|^{2} .
$$

Since $\left|g_{y}(t, y)\right| \leq 4$, choosing $\left|\gamma_{n}\right| \geq \sqrt{2 R e}$, i.e.,

$$
|n| \geq M=\frac{2 h \sqrt{R e}}{\pi}
$$

yields

$$
\frac{d}{d t}\left\|\mathbf{w}_{n}\right\|^{2} \leq-2 \frac{\left\|\mathbf{w}_{n y}\right\|^{2}}{R e}-\gamma_{n}^{2} \frac{\left\|\mathbf{w}_{n}\right\|^{2}}{R e} \leq-2 \frac{\left\|\mathbf{w}_{n}\right\|^{2}}{R e},
$$

by Poincare's inequality, therefore achieving $L^{2}$ exponential stability for large modes $(|n| \geq M)$.

\subsection{Controlled modes. Construction of control laws}

The remaining modes, $0<|n|<M$, are open-loop unstable and must be controlled. We design the control in several steps.

\subsubsection{Pressure shaping}

Solving (80)-(82),

$$
\begin{aligned}
p_{n}= & -2 i \int_{0}^{y} g_{y}(t, \eta) \sinh \left(\gamma_{n}(y-\eta)\right) v_{n}(t, \eta) d \eta \\
& +2 i \frac{\cosh \left(\gamma_{n} y\right)}{\sinh \gamma_{n}} \int_{0}^{1} g_{y}(t, \eta) \cosh \left(\gamma_{n}(1-\eta)\right) v_{n}(t, \eta) d \eta \\
& +i \frac{\cosh \left(\gamma_{n}(1-y)\right)}{\sinh \gamma_{n}} \frac{u_{n y}(0, t)}{R e}-\frac{\left.\cosh \left(\gamma_{n} y\right)\right)}{\sinh \gamma_{n}}\left(i \frac{u_{n y}(1, t)}{R e}+\frac{\dot{V}_{n}}{\gamma_{n}}\right. \\
& \left.+\gamma_{n} \frac{V_{n}}{R e}\right) .
\end{aligned}
$$

Note that $V_{n}$ appears in (90), allowing to "shape" it. We design $V_{n}$ to enforce in (90) a strict-feedback structure [KRS 95] in $y$. This structural property is a sort of "spatial causality", which requires that in the expression for, say, $f_{t}(y)$, no value of $f(s)$ for $s>y$ appears. It is a technical requirement in the backstepping method for parabolic equations [SMY 04, SMY 05b], which we use next. Seeking the strictfeedback structure in (90), we choose $V_{n}$ as

$$
\begin{aligned}
\frac{\dot{V}_{n}}{\gamma_{n}}= & -\gamma_{n} \frac{V_{n}}{R e}+i \frac{u_{n y}(0, t)-u_{n y}(1, t)}{R e}-2 i \int_{0}^{1} g_{y}(t, \eta) \cosh \left(\gamma_{n}(1-\eta)\right) \\
& \times v_{n}(t, \eta) d \eta .
\end{aligned}
$$


Introducing (91) into (90), and (90) into (74)-(75), yields

$$
\begin{aligned}
u_{n t}= & \frac{u_{n y y}}{R e}-\frac{\gamma_{n}^{2} u_{n}}{R e}+i \gamma_{n} g(t, y) u_{n}+g_{y}(t, y) v_{n} \\
& +2 \gamma_{n} \int_{0}^{y} g_{y}(t, \eta) \sinh \left(\gamma_{n}(y-\eta)\right) v_{n}(t, \eta) d \eta \\
& +\gamma_{n} \frac{\left.\cosh \left(\gamma_{n}(1-y)\right)-\cosh \left(\gamma_{n} y\right)\right)}{\operatorname{Resinh} \gamma_{n}} u_{n y}(0), \\
v_{n t}= & \frac{v_{n y y}}{R e}-\frac{\gamma_{n}^{2} v_{n}}{R e}+i g(t, y) v_{n} \\
& -2 i \gamma_{n} \int_{0}^{y} g(t, \eta) \cosh \left(\gamma_{n}(y-\eta)\right) v_{n}(t, \eta) d \eta \\
& +i \gamma_{n} \frac{\left.\sinh \left(\gamma_{n}(1-y)\right)+\sinh \left(\gamma_{n} y\right)\right)}{\operatorname{Re} \sinh \gamma_{n}} u_{n y}(0) .
\end{aligned}
$$

\subsubsection{Control of velocity field}

Our objective is now to control (92)-(93) by means of $U_{n}$. By (79), $v_{n}$ can be computed from $u_{n}$. Then, only (92) has to be considered. Using (79) to express (92) as an autonomous equation in $u_{n}$,

$$
\begin{aligned}
u_{n t}= & \frac{\triangle_{n} u_{n}}{R e}+\lambda_{n}(t, y) u_{n}+\int_{0}^{y} f_{n}(t, y, \eta) u_{n}(t, \eta) d \eta \\
& +h_{n}(y) u_{n y}(0, t),
\end{aligned}
$$

with boundary conditions

$$
\begin{aligned}
& u_{n}(t, 0)=0, \\
& u_{n}(t, 1)=U_{n}(t),
\end{aligned}
$$

where $\lambda_{n}, f_{n}$ and $h_{n}$ were defined in (30)-(32). This is a boundary control problem for a parabolic PIDE with time-dependent coefficients, solvable by backstepping [SMY 05b] thanks to the strict-feedback structure. Following [SMY 05b] we map $u_{n}$, for each mode $0<|n|<M$, into the family of heat equations:

$$
\begin{aligned}
\alpha_{n t} & =\frac{1}{R e}\left(-\gamma_{n}^{2} \alpha_{n}+\alpha_{n y y}\right) \\
\alpha_{n}(k, 0) & =\alpha_{n}(k, 1)=0,
\end{aligned}
$$

where

$$
\begin{aligned}
& \alpha_{n}=\left(I-K_{n}\right) u_{n} \\
& u_{n}=\left(I+L_{n}\right) \alpha_{n},
\end{aligned}
$$


are respectively the direct and inverse transformation. The kernel $K_{n}$ is found to verify equations (27)-(29), and $L_{n}$ verifies a similar equation, or can be derived from $K_{n}$ using Proposition 5.1. For (27)-(29), the following result holds.

Proposition 6.1 There exists a bounded solution $K_{n}$ of (27)-(29) defined in $\Gamma, \mathcal{C}^{1}$ in space and $\mathcal{C}^{\omega}$ in time.

Proof We sketch a proof following [SMY 04, COL 77, SMY 05b], where similar (though simpler) equations were studied.

Mirroring [SMY 04], we transform (27)-(29) to an integral-differential equation. Analyticity ${ }^{5}$ and uniform boundedness in time and smoothness in space of the coefficients allows to construct a solution $K_{n}^{*}$ defined for $t \in J^{*} \subset(0, \infty)$, where $J^{*}$ is open and finite ${ }^{6}$, by using a domination method [COL 77]. Since the dominating function is independent of $J^{*}$, all such $K_{n}^{*}$ are uniformly bounded in $t$, and a classical argument [RUD 86] shows these $K_{n}^{*}$ to be $\mathcal{C}^{1}$ in space and $\mathcal{C}^{\omega}$ in time.

Consider now a collection $J^{i}$ of open finite intervals, such that $\bigcup J^{i} \subseteq(0, \infty)$ and ordered such that $J^{i} \cap J^{i+1} \neq \emptyset$. Following the previous paragraph, we construct solutions $K_{n}^{i}$ defined for $t \in J^{i}$. Now, it is possible to show that in any open time interval (27)-(29) only admits one analytic solution. Therefore, in $J^{i} \cap J^{i+1}$, $K_{n}^{i} \equiv K_{n}^{i+1}$. This allows gluing together the solutions $K_{n}^{i}$ to obtain a solution $K_{n}$ defined in the whole $\Gamma$, which by construction is $\mathcal{C}^{1}$ in space and $\mathcal{C}^{\omega}$ in time. Uniform boundedness in $t$ of the family $K_{n}^{i}$ implies the boundedness of the glued solution $K_{n}$.

The control law is, from (99), (98) and (96)

$$
U_{n}=\int_{0}^{1} K_{n}(t, 1, \eta) u_{n}(t, k, \eta) d \eta
$$

Stability properties of the closed loop system follow from (97)-(98) and (99)(100). We obtain

$$
\frac{d}{d t}\left\|u_{n}\right\|_{K_{n} L^{2}(0,1)}^{2} \leq \mathrm{e}^{-\frac{2}{R e} t}\left\|u_{n}(0)\right\|_{K_{n} L^{2}(0,1)}^{2}
$$

5. This requirement, which we verify, cannot be easily dropped [KAN 90].

6 . In fact one must consider $t$ a complex variable defined in a region that contains the positive real axis, e.g. a semi-infinite strip. We skip the details. 


\subsection{Stability for the whole system}

If we call $A=\{n \in \mathbb{Z}: 0<|n|<M\}$, and $\mathcal{K}=K_{n}(t, y, \eta)_{n \in A}$, and apply the control laws (101)-(91) in physical space, which yield (26)-(25), then we can prove stability in the $\mathcal{K} H_{0 h}(\Omega)$ norm.

$$
\begin{aligned}
\|\mathbf{w}\|_{\mathcal{K} H_{0 h}(\Omega)}^{2} & =\sum_{n \notin A}\left\|\mathbf{w}_{n}\right\|_{L^{2}(0,1)^{2}}^{2}+\sum_{n \in A}\left\|u_{n}\right\|_{K_{n} L^{2}(0,1)}^{2} \\
& \leq \mathrm{e}^{-\frac{2}{R e} t}\left[\sum_{n \notin A}\left\|\mathbf{w}_{n}(0)\right\|_{L^{2}(0,1)^{2}}^{2}+\sum_{n \in A}\left\|u_{n}(0)\right\|_{K_{n} L^{2}(0,1)}^{2}\right] \\
& \leq \mathrm{e}^{-\frac{2}{R e} t}\|\mathbf{w}(0)\|_{\mathcal{K} H_{0 h}(\Omega)}^{2},
\end{aligned}
$$

and by norm equivalency, this proves Theorem 4.1.

\section{Proof of theorem 4.2}

We now consider the full Navier-Stokes equation. Let us call the nonlinear term $\mathbf{N}=\left(N^{u}, N^{v}\right)$, where

$$
\begin{aligned}
& N^{u}=-u u_{x}-v u_{y} \\
& N^{v}=-u v_{x}-v v_{y}
\end{aligned}
$$

One has that

$$
\begin{aligned}
(\mathbf{w}, \mathbf{N})= & \left(u, N^{u}\right)+\left(v, N^{v}\right) \\
= & \left(u, u u_{x}\right)+\left(v, u v_{x}\right)+\left(u, v u_{y}\right)+\left(v, v v_{y}\right) \\
\leq & \frac{1}{c}\left[\left\|u_{x}\right\|^{2}+\left\|u_{y}\right\|^{2}+\left\|v_{x}\right\|^{2}+\left\|v_{y}\right\|^{2}\right] \\
& +\frac{c}{4}\left[\left(u^{2}, u^{2}\right)+2\left(v^{2}, u^{2}\right)+\left(v^{2}, v^{2}\right)\right],
\end{aligned}
$$

for any $c>0$, where we applied Young's Inequality. By Lemma 5.1, and using norm equivalences, one then has

$$
(\mathbf{w}, \mathbf{N})_{H_{0 h}(\Omega)} \leq c_{1}\|\mathbf{w}\|_{\mathcal{K} H_{1 h}(\Omega)}^{2}+\frac{C_{1}}{c_{1}}\|\mathbf{w}\|_{\mathcal{K} H_{0 h}(\Omega)}^{2}\|\mathbf{w}\|_{\mathcal{K} H_{1 h}(\Omega)}^{2},
$$

for $c_{1}$ positive to be chosen, and some $C_{1}>0$. The bound above is valid not only for $(\mathbf{w}, \mathbf{N})$ but for any partial sum of $\left(\mathbf{w}_{n}, \mathbf{N}_{n}\right)$, by the same argument.

The application of pressure shaping and backstepping transformation to the nonlinear system results in a new term in the target system, which appears as

$$
\alpha_{n t}=\frac{1}{R e}\left(-\gamma_{n}^{2} \alpha_{n}-\alpha_{n y y}\right)+N_{n}^{\alpha}
$$


where the definition of $N_{n}^{\alpha}$ is

$$
N_{n}^{\alpha}=\left(I-K_{n}\right) N_{n}^{u}+\left(I-K_{n}\right) N_{n}^{p} .
$$

The term $N_{n}^{p}$ is due to pressure shaping and is defined as

$$
\begin{aligned}
N_{n}^{p}= & \frac{2}{\gamma_{n}} \sum_{j \in \mathbb{Z}}\left[\frac{\cosh \left(\gamma_{n} y\right)}{\sinh \gamma_{n}} \int_{0}^{1} N_{n j}^{q} \cosh \left(\gamma_{n}(1-\eta)\right) d \eta\right. \\
& \left.+\int_{0}^{y} N_{n j}^{q} \sinh \left(\gamma_{n}(y-\eta)\right) d \eta\right],
\end{aligned}
$$

where

$$
N_{n j}^{q}=\gamma_{j} \gamma_{n-j} u_{j} u_{n-j}+\gamma_{n-j} u_{j y} v_{n-j} .
$$

Then, for $n \in A$,

$$
\begin{aligned}
\left(\alpha_{n}, N_{n}^{\alpha}\right) \leq & C_{2}\left[\left(\left|\alpha_{n}\right|,\left|N_{n}^{u}\right|\right)+\left(\left|\alpha_{n}\right|,\left|N_{n}^{p}\right|\right)\right] \\
\leq & C_{2}|| \alpha_{n}|| \sum_{j \in \mathbb{Z}}\left\{\left[|| \gamma_{j}||\left|u_{j} u_{n-j}\right| \mid\right.\right. \\
& \left.\left.+\| u_{j y} v_{n-j}||\right]\left[1+C_{3}\left|\gamma_{n-j}\right|\right]\right\}
\end{aligned}
$$

where $C_{2}=1+\|\mathcal{K}\|_{\infty}$ and $C_{3}=\frac{\sinh \left(\gamma_{1}\right)+\cosh ^{2}\left(\gamma_{M}\right)}{\sinh \left(\gamma_{1}\right) \gamma_{1}}$. Bounding the previous expression further, one gets

$$
\begin{aligned}
\left(\alpha_{n}, N_{n}^{\alpha}\right) \leq & \frac{C_{2}}{2}\left\|\alpha_{n}\right\| \sum_{j \in \mathbb{Z}}\left\{\left|\gamma_{j}\right|^{2}\left\|u_{j}\right\|^{2}+\left\|u_{j y}\right\|^{2}\right. \\
& {\left.\left[1+C_{3}^{2}\left|\gamma_{n-j}\right|^{2}\right]\left[\left\|u_{(n-j)}\right\|^{2}+\left\|v_{(n-j)}\right\|^{2}\right]\right\} } \\
\leq & C_{4}\left\|\alpha_{n}\right\|\|\mathbf{w}\|_{\mathcal{K}_{H}(\Omega)}^{2},
\end{aligned}
$$

for some positive $C_{4}$. Calculate now the $\mathcal{K} L^{2}$ norm of the controlled Navier-Stokes equation. As before:

$$
\|\mathbf{w}\|_{\mathcal{K} L_{0 h}^{2}(\Omega)}^{2}=\sum_{n \notin A}\left\|\mathbf{w}_{n}\right\|+\sum_{n \in A}\left\|u_{n}\right\|_{K_{n} L^{2}(0,1)}^{2} .
$$

Calculate now the derivatives for all this terms.

$$
\frac{d}{d t} \sum_{n \notin A}\left\|\mathbf{w}_{n}\right\|^{2} \leq \sum_{n \notin A}\left[\frac{-2}{R e}\left\|\mathbf{w}_{n y}\right\|^{2}-\frac{\gamma_{n}^{2}}{R e}\left\|\mathbf{w}_{n}\right\|^{2}+\left(\mathbf{w}_{n}, \mathbf{N}_{n}\right)\right],
$$

and for $n \in A$, since $\left\|u_{n}\right\|_{K_{n} L^{2}(0,1)}=\left\|\alpha_{n}\right\|_{L^{2}(0,1)}$,

$$
\frac{d}{d t}\left\|\alpha_{n}\right\| \leq \frac{-2}{R e}\left\|\alpha_{n y}\right\|+\frac{-2 \gamma_{n}^{2}}{R e}\left\|\alpha_{n}\right\|+\left(\alpha_{n}, N_{n}^{\alpha}\right) .
$$


Then, summing (116) for $n \in A$, adding (115), and applying norm equivalences, we get for some $C_{0}>0$

$$
\begin{aligned}
\frac{d}{d t}\|\mathbf{w}\|_{\mathcal{K} H_{0 h}(\Omega)}^{2} \leq & -C_{0}\|\mathbf{w}\|_{\mathcal{K}_{1 h}(\Omega)}^{2}+\sum_{n \notin A}\left(\mathbf{w}_{n}, \mathbf{N}_{n}\right) \\
& +\sum_{n \in A}\left(\alpha_{n}, N_{n}^{\alpha}\right)_{L^{2}(0,1)} \\
\leq & \|\mathbf{w}\|_{\mathcal{K} H_{1 h}(\Omega)}^{2}\left(C_{4}\|\mathbf{w}\|_{\mathcal{K} H_{0 h}(\Omega)}\right. \\
& \left.+c_{1}+\frac{C_{1}}{c_{1}}\|\mathbf{w}\|_{\mathcal{K} H_{0 h}(\Omega)}^{2}-C_{0}\right) .
\end{aligned}
$$

Choose $c_{1}=C_{0} / 2$, and suppose $\|\mathbf{w}\|_{\mathcal{K} H_{0 h}(\Omega)}<\epsilon$. Then

$$
\frac{d}{d t}\|\mathbf{w}\|_{\mathcal{K} H_{0 h}(\Omega)}^{2} \leq\left(\frac{2 C_{1}}{C_{0}} \epsilon^{2}+C_{4} \epsilon-\frac{C_{0}}{2}\right)\|\mathbf{w}\|_{\mathcal{K} H_{1 h}(\Omega)}^{2},
$$

and choosing $\epsilon$ such that $2 C_{1} / C_{0} \epsilon^{2}+C_{4} \epsilon<\frac{C_{0}}{4}$,

$$
\begin{aligned}
\frac{d}{d t}\|\mathbf{w}\|_{\mathcal{K} H_{0 h}(\Omega)}^{2} & \leq \frac{-C_{0}}{4}\|\mathbf{w}\|_{\mathcal{K} H_{1 h}(\Omega)}^{2} \\
& \leq-C_{5}\|\mathbf{w}\|_{\mathcal{K} H_{0 h}(\Omega)}^{2},
\end{aligned}
$$

by applying an equivalent version of Poincare's inequality, where $C_{5}>0$. This proves local exponential stability in the $\mathcal{K} H_{0 h}(\Omega)$ norm and therefore in the $H_{0 h}(\Omega)$ norm.

\section{Acknowledgements}

We thank T. Bewley and M. Krstic for suggesting the approach of Section 3, and A. Smyshlyaev for helpful discussions regarding kernel equations (27)-(29).

\section{References}

[BAT 67] Batchelor G., An Introduction to Fluid Mechanics, Cambridge University Press, London, 1967.

[COL 77] COLTON D., "The solution of initial-boundary value problems for parabolic equations by the method of integral operators", Journal of Differential Equations, vol. 26, 1977, p. $181-190$.

[COR 02] CORON J., "Local controllability of a 1D tank containing a fluid modeled by the shallow water equations", ESAIM: Contr. Optim. Calc. Variat., vol. 8, 2002, p. 513-554.

[COR 04] CORON J., TRÉlat E., "Global steady-state controllability of 1D semilinear heat equations", SIAM J. Control and Optimization, vol. 43, 2004, p. 549-569. 
[COR 05] CORON J., TRÉlat E., "Global steady-state stabilization and controllability of 1D semilinear wave equations”, , 2005, Preprint.

[EVA 98] Evans L., Partial Differential Equations, American Mathematical Society, Providence, Rhode Island, 1998.

[HOC 73] Hochstadt H., Integral Equations, Wiley, New York, 1973.

[HOE 05] Hoepffner J., Chevalier M., Bewley T., Henningson D., "State estimation in wall-bounded flow systems. Part 1. Perturbed laminar flows", Journal of Fluid Mechanics, vol. to appear, 2005.

[HOG 03] Hogberg M., Bewley T., Henningson D., "Linear feedback control and estimation of transition in plane channel flow", Journal of Fluid Mechanics, vol. 481, 2003, p. 149-175.

[KAN 90] KANNAI Y., "Nonexistence for a boundary value problem arising in parabolic theory", Israel Journal of Mathematics, vol. 71, 1990, p. 349-351.

[KER 82] KERCZEK C. V., "The instability of oscillatory plane Poiseuille flow", Journal of Fluid Mechanics, vol. 116, 1982, p. 91-114.

[KRS 95] Krstic M., Kanellakopoulos I., Kokotovic P. V., Nonlinear and Adaptive Control Design, Wiley, New York, 1995.

[RUD 86] RUdin W., Real and Complex Analysis, Mc. Graw-Hill, New York, 1986.

[SCH 01] SCHMID P., Henningson D., Stability and Transition in Shear Flows, Springer, New York, 2001.

[SCH 05] SCHMIDT M., TRÉlAT E., "Controllability of Couette-Taylor flows by rotating cylinders", Comm. Pure Applied Analysis, vol. To appear, 2005.

[SMY 04] SMYSHLYAEV A., KRSTIC M., "Closed form boundary state feedbacks for a class of partial integro-differential equations", IEEE Transactions on Automatic Control, vol. 49, 2004, p. 2185-2002.

[SMY 05a] SMYShlYAEV A., KRstic M., "Backstepping observers for parabolic PDEs", Systems and Control Letters, vol. 54, 2005, p. 1953-1971.

[SMY 05b] SMYshlyaev A., KRStic M., "On control design for PDEs with spacedependent diffusivity or time-dependent reactivity", Automatica, vol. 41, 2005, p. 16011608.

[TEM 84] Temam R., Navier-Stokes Equations, Theory and Numerical Analysis, NorthHolland Publishing Co., Amsterdam, 1984.

[V’05a] VÁzquez R., KRstic M., "A closed-form feedback controller for stabilization of linearized Navier-Stokes equations: the 2D Poisseuille flow", Conference on Decision and Control, 2005.

[V’05b] VÁzquez R., KRstic M., “A closed-form observer for the channel flow NavierStokes system”, Conference on Decision and Control, 2005. 